\title{
16th Topical Meeting on the Optics of Liquid Crystals
}

\author{
Janusz Parka $^{* 1,2}$ and Stanisław Kłosowicz ${ }^{* 1}$ \\ ${ }^{I}$ Institute of Applied Physics, Military University of Technology, Warsaw, Poland \\ ${ }^{2}$ Institute of Microelectronics and Optoelectronics, Warsaw University of Technology
}

Received December 30, 2015; accepted December 30, 2015; published December 31, 2015

\begin{abstract}
The current issue of the Photonics Letters of Poland contains 5 selected papers presented during the 16th Topical Meeting on the Optics of Liquid Crystals, which was held from September 13 to 18, 2015 in Sopot, Poland. The remaining 8 papers included in the issue are regular contributions.
\end{abstract}

The Optics of Liquid Crystals 2015 (OLC 2015) was held in Sopot, Poland, from September 13 to 18. The venue of the conference was the charming and welcoming Grand Hotel Sopot, situated at a pleasant beach of the Baltic Sea.

The conference series has been organized every two years since 1986. The first one was in Naples (Italy) and two years ago - in Honolulu (USA). In 2014 the International Advisory Board decided to entrust Poland with the task of organizing the OLC 2015. The conference was organized by the Military University of Technology (financial support) and the Warsaw University of Technology and held under the auspices of the Polish Liquid Crystal Society and the Photonics Society of Poland.

Timothy Bunning (Georgia Institute of Technology, USA), Chairman of the OLC Advisory Board opened the conference with a talk about dynamic optical polymer/liquid crystalline systems. The level of the talks and poster presentations was very high, giving participants an excellent and broad overview of research activities in the optics of liquid crystals (LCs).

About 160 attendees from Poland, Germany, Belarus, Ukraine, France, Hungary, Sweden, Spain, Belgium, UK, Italy, Japan, USA, South Korea, Canada, Brazil, Mexico, India, China, Taiwan, and Saudi Arabia participated in the OLC 2015. The scientific program was highly condensed (without parallel sections) including 13 invited talks, 51 oral presentations, 70 posters, as well 4 tutorial lectures.

The conference topics comprised the optics of complex anisotropic structures, nonlinear optics, 3D optics, photonics and displays, applications of blue phase materials and devices, novel LC optical materials, LCs in high power laser systems, diffractive waveplates, polarization gratings, holographic systems, laser beam steering and optical switching, optical and photonic properties enhanced by surfaces and interfaces,

\footnotetext{
*E-mail: janusz.parka@wat.edu.pl, stanislaw.klosowicz@wat.edu.pl
}

photoalignment techniques and materials, photovoltaics and solitons in anisotropic materials, LC lenses, optics of LCs doped with nanoparticles and nanowires.

Three best student presentations were awarded during the OLC 2015: Inge Nys (Ghent Univ., Belgium): "3D LC grating resulting from periodic photo-alignment at both substrates", Markus Wahle (Univ. of Paderborn, Germany): "Electrically tunable waveguide properties in hybrid guiding LC photonic crystal fibers", and Jae-Won Huh (Pusan National Univ., South Korea): "Switching of dye-doped long-pitch cholesteric CLCs for light shutter application".

The social program of the conference included a visit to the famous Oliva Church, where an exquisite organ concert took place. After the church there was an excursion to the historical Old City of Gdańsk and to the Museum of Solidarity, a witness of the civil resistance of the Polish shipyard workers in 1980. The atmosphere of the conference and its organization were outstanding.

In this issue of the Photonics Letters of Poland we include 5 papers which were presented during the OLC 2015 conference. The first contribution of S. Kredentse $e t$ al. is devoted to dynamic and permanent gratings in suspensions of absorbing nanocrystals in organic solvent mince nematic LCs. The Soret effect described in this paper gives an opportunity to apply light-induced gratings to elaborate highly-sensitive non-linear optical media. The second paper (Y.G. Marinov et al.) was devoted to thin films of silica nanoparticle doped to 7CB nematic LC for electro-optic second-harmonic EO modulation. Another paper (S. Pandey et al.) describes the analysis of faster optical response in core/shell nanocrystals ferroelectric LC composite systems for faster electro-optical devices. P. Wierzba and A. Mazikowski reported on a polarization interferometer with an LC polymer waveplate, whereas G. Derfel and M. Buczkowska discussed the application of twisted nematics cells in beam steering.

The other 8 papers in this volume are regular contributions devoted to other fields of photonics.

This editorial is a good opportunity to thank all the members of the Scientific Committee for their efforts in assuring a high scientific level of the conference. Also, we would like to express our gratitude to all the participants of the meeting. 